Original Article

\title{
Clinical Presentation and Surgical Outcomes of Chronic Subdural Hematoma
}

\author{
Mukhtiar Ahmed', Fauzia Sajjad², Ajmal Khan ${ }^{3}$, Talha Abass ${ }^{4}, H_{a m i d ~ A k b a r}{ }^{2}$ \& Khawar Anwar \\ ${ }^{1}$ Department of Neurosurgery Khairpur Medical College, Sindh, Pakistan. \\ ${ }^{2}$ Punjab Institute of Neurosciences (PINS), ${ }^{3}$ Services Hospital, ${ }^{4}$ Fatima Jinnah Medical University, Lahore, \\ Pakistan. ${ }^{5}$ Department of Neurosurgery, Peoples Medical University of Health Sciences (PMUHS), District \\ Shaheed Benazir Abad, Pakistan
}

\begin{abstract}
Objective: The aim of this study was to access clinical presentation of chronic subdural hematoma and to evaluate the surgical outcome of evacuation of chronic subdural hematoma after single burrhole craniotomy with a close drainage system under local anesthesia.
\end{abstract}

Material \& Methods: A descriptive study conducted in the Neurosurgery Department of Khairpur Medical College Sindh. 30 patients of chronic subdural hematoma were included. Patients prepared for evacuation of chronic subdural hematoma through single burr hole followed by closed drainage system for two days.

Results: The most common feature was gait disturbance followed by Hemiparesis and headache. Coexisting diseases were hypertension, Diabetes mellitus and Chronic Liver Disease, etc. and causes of CSDH were head injury (trivial trauma) and anticoagulant. Surgical decisions were taken on the bases of clinical presentation and radiological findings of patients. Surgical procedure single burrhole craniotomy followed by irrigation with normal saline and close drainage system for two days. On post-operative follow-up, a recurrence of CSDH occurred in two patients.

Conclusion: Single burrhole craniotomy with close drainage system is a simple and safe procedure under local anesthesia for treatment of chronic subdural hematoma.

Keywords: CSDH (chronic subdural hematoma), GCS (Glasgow coma score), Burrhole craniotomy, Neurosurgery \& Subdural drain.

Corresponding Author: Mukhtiar Ahmed

Department of Neurosurgery Khairpur Medical College

Sindh

Email: ahmed.mukhtiar77@gmail.com

Date of Submission: 9-11-2020

Date of Revision: 20-12-2020

Date of Online Publishing: 25-12-2020

Date of Print: $30-12-2020$
DOI: $10.36552 /$ pjns.v24i4.502

\section{INTRODUCTION}

Chronic subdural hematoma (CSDH) is a common clinical entity in neurosurgical department. It mainly occurs in old age people few weeks after head injury. The annual incidence of chronic 
subdural hematoma has been reported to be 1 5.3 cases per 100000 populations. ${ }^{1}$ The aim of this study is to access clinical presentation of chronic subdural hematoma and to evaluate the surgical outcome of evacuation of chronic subdural hematoma after single burrhole craniotomy with a close drainage system under local anesthesia. Chronic subdural hematoma may present with headache, vomiting abnormal behavior and focal neurological deficit for example altered conscious level, hemiplegia, and dysphasia, seizures (focal or generalized). ${ }^{2}$ Chronic subdural hematoma may continue to increase with time, which suggesting acute hematoma is not the only source of increase size of chronic subdural hematoma. ${ }^{3}$ Chronic subdural hematoma diagnosed by CT scan brain. Hematoma appears as crescentic shape hypo dense lesions causing compression of the ipsilateral cerebral hemisphere and obliteration of ipsilateral ventricles. Bilateral isodense chronic subdural hematoma may be difficult to diagnose by the CT scan brain. MRI could be helpful in diagnosis of isodense chronic subdural hematoma. ${ }^{4} \quad$ Chronic subdural hematoma may be an asymptotic but in case of symptoms, surgical treatment is required. Brain atrophy is common in old age and causing traction on bridging veins. So, even trivial trauma may increase the risk of tearing of bridging veins into subdural space. ${ }^{5}$ Chronic subdural hematoma (CSDH) may cause serious morbidity and mortality particularly in elderly people.

Surgical evacuation is the treatment of choice for symptomatic patients. Burrhole craniotomy and the close drainage system is worldwide used technique for management of $\mathrm{CSDH}^{6}{ }^{6}$ Surgical evacuation of the hematoma was done when following radiological criteria was observed; unilateral chronic subdural hematoma thickness is more than $10 \mathrm{~mm}$. Midline shift more than $5 \mathrm{~mm}$, mass effect, neurologic sign and symptoms due to increased intra cranial pressure and Progressive increase in size of CSDH on serial CT scan of brain. ${ }^{7}$ The current study aimed to access clinical presentation of chronic subdural hematoma and to evaluate the surgical outcome of evacuation of chronic subdural hematoma after single burrhole craniotomy with a close drainage system under local anesthesia.

\section{MATERIAL AND METHODS}

\section{Study Design and Setting}

It was descriptive study conducted at the Department of Neurosurgery of Khairpur Medical College, Sindh from October 2016 to October 2019. All Patients of chronic subdural hematoma were admitted in the Neurosurgery department of Khairpur Medical College Sindh were included.

\section{Inclusion Criteria}

Progressive neurological deficit and chronic subdural hematoma with midline shift more than $5 \mathrm{~mm}$ on CT scan brain plain.

\section{Exclusion Criteria}

Recurrent chronic subdural hematoma, chronic subdural hematoma with bleeding disorder.

\section{Sampling}

Krejcie and Morgan, (1973) table was used to set sample size and 30 patients of chronic subdural hematoma were included after exclusion criteria in this study during the period of 2016 to 2019.

\section{Data Collection}

The study was carried on 30 patients having age of between $50-80$ years. The basic demographic information was recorded. A neurological clinical assessment was done at time of admission. ${ }^{8} \mathrm{CT}$ scan brain chronic subdural hematoma was assessed for the thickness of hematoma, along with its density, volume \& midline shift. Baseline investigations before surgery included complete blood count, pro-thrombin time (PT) and 
activated partial thromboplastin time (APTT), international normalized ratio (INR), serum electrolytes, blood urea and creatinine.

Symptomatic patients with neurological deficit and CT scan brain has shown a midline shift more than $5 \mathrm{~mm}$, volume of hematoma more than $25 \mathrm{ml}$, and hematoma thickness more than $15 \mathrm{~mm}$ prepared for surgery. Under local anesthesia and sedation, the head tilted to contra-literal side than single parietal burr hole made. Dura coagulated and opened in cruciate manner dural edges coagulate up to bone ages, hematoma evacuated and cavity washed and filled with normal saline, closed subdural drainage system place for two days. Post operatively, the patient kept in a supine position. Drain removed on the second post-operative day.

\section{Data Analysis}

All results were taken through SPSS 23.

\section{RESULTS}

\section{Age Incidence}

Age range was $50-80$ years.

\section{Gender Distribution}

There were 25 male patients and 5 female patients.

Chronic subdural hematoma was diagnosed by CT scan brain in all patients. CSDH located on right side in 15 patients, on left side in 9 patients and bilateral cases were 6 out of 30. Hematoma thickness was found between 15 to $30 \mathrm{~mm}$ right and in left sides, 20 to $40 \mathrm{~mm}$ in cases of bilateral $\mathrm{CSDH}$. The volume of hematoma in these cases was 20 to $70 \mathrm{cc}$ on left side, 25 to $60 \mathrm{cc}$ on right side and $40 \mathrm{cc}$ to $80 \mathrm{cc}$ on bilateral. Table 1 representing density of $\mathrm{CSDH}$ hematoma in 30 cases, it was hypo density in $50 \%$ cases, isodensity in $40 \%$, and mixed density $10 \%$.
Table 1: Characteristics of 30 Patients with CSDH

\begin{tabular}{|c|c|c|}
\hline \multicolumn{3}{|c|}{ Hematoma Location } \\
\hline Left & Right & Bilateral \\
\hline 9 & 15 & 6 \\
\hline \multicolumn{3}{|c|}{ Hematoma Thickness } \\
\hline Left & Right & Bilateral \\
\hline 15 to $30 \mathrm{~mm}$ & 15 to $30 \mathrm{~mm}$ & 20 to $40 \mathrm{~mm}$ \\
\hline \multicolumn{3}{|c|}{ Volume of Hematoma } \\
\hline Left & Right & Bilateral \\
\hline 20 to $70 \mathrm{cc}$ & 25 to $60 c c$ & 40 to 80 \\
\hline \multicolumn{3}{|c|}{ Hematoma Density } \\
\hline Hypodensity & Isodensity & Mixed density \\
\hline $50 \%$ & $40 \%$ & $10 \%$ \\
\hline
\end{tabular}

\section{Co-Morbidities}

Table 2 showing the coexisting disease in patients with $\mathrm{CSDH} .9$ patients were suffered from Hypertension, 5 patients were having Diabetes mellitus, 3 patients were taking Anti-coagulant drugs, 2 patients were the cases of Chronic Liver Disease and 1 patient was with the cerebral infarction.

\begin{tabular}{lc|}
\hline Table 2: Coexisting diseases in 30 patients with \\
CSDH. & No. of Patients \\
Coexisting Disease & 1 \\
Cerebral infarction & 9 \\
Hypertension & 2 \\
Chronic Liver Disease & 3 \\
Anti-coagulant drugs & 5 \\
Diabetes mellitus &
\end{tabular}

\section{Clinical Presentation of CSDH}

Table 3 showing clinical presentation of $\mathrm{CSDH}$, mostly patient presented with Gait disturbance and one side weakness (Hemiparesis) was the

Table 3: Clinical Presentation of CSDH.

\begin{tabular}{lc} 
Clinical Presentation & No. of Patients \\
Gait disturbance & 17 \\
Hemiparesis & 17 \\
Headache & 11 \\
Dementia & 10 \\
Incontinence & 7 \\
\hline
\end{tabular}




\begin{tabular}{|ll|}
\hline Altered Conscious level & 6 \\
Vomiting & 3 \\
Seizures & 3 \\
Motor aphasia & 2 \\
\hline
\end{tabular}

main finding. The 11 patients reported with headache, 10 patients suffered from dementia, incontinence was found in 7 patients, altered conscious level was observed in 6 patients, 3 patients reported vomiting, and seizures were found in 3 patients and 2 patients suffered from the speech problem.

\section{CSDH and Head Injury}

Table 4 is representing the CSDH caused by mild head injury (trivial trauma) in 17 patients, anticoagulant in 6 patients and unknown causes were found in 9 patients in the study.

Table 4: Causes of CSDH.

\section{Cause}

Head injury

Anti-coagulant

Unknown

\section{No. of Patients}

17

6

9

\section{GCS at the Time of Admission}

At the time of admission, the patients' GCS was checked (Table 5). 5 patients had GCS between 5 to 8,11 patients GCS 9 to 12,13 patients GCS 13 to 14 and GCS 15 was found in only 1 patient.

Table 5: Immediate pre-operative status.

\section{Pre-operative GCS}

5 to 8

9 to 12

13 to 14

15

No of patients
5
11
13
1

\section{Postoperative GCS}

After surgical evacuation of chronic subdural hematoma, the postoperative GCS was improved in all patients. In postoperative follow-up, the recurrence of subdural hematoma seen in two patients, fortunately no any patient suffered from tension pneumocephalus because cavity filled with normal saline after evacuation of subdural hematoma.

About three weeks before, a patient with mild head injury was presented with left side weakness and headache in neurosurgery department. After radiological work up, the $\mathrm{CT}$ brain plain showing right fronto parietal chronic subdural hematoma with an acute component and compression of ipsilateral ventricles with mid line shift to contra lateral side of brain (See figure 1).

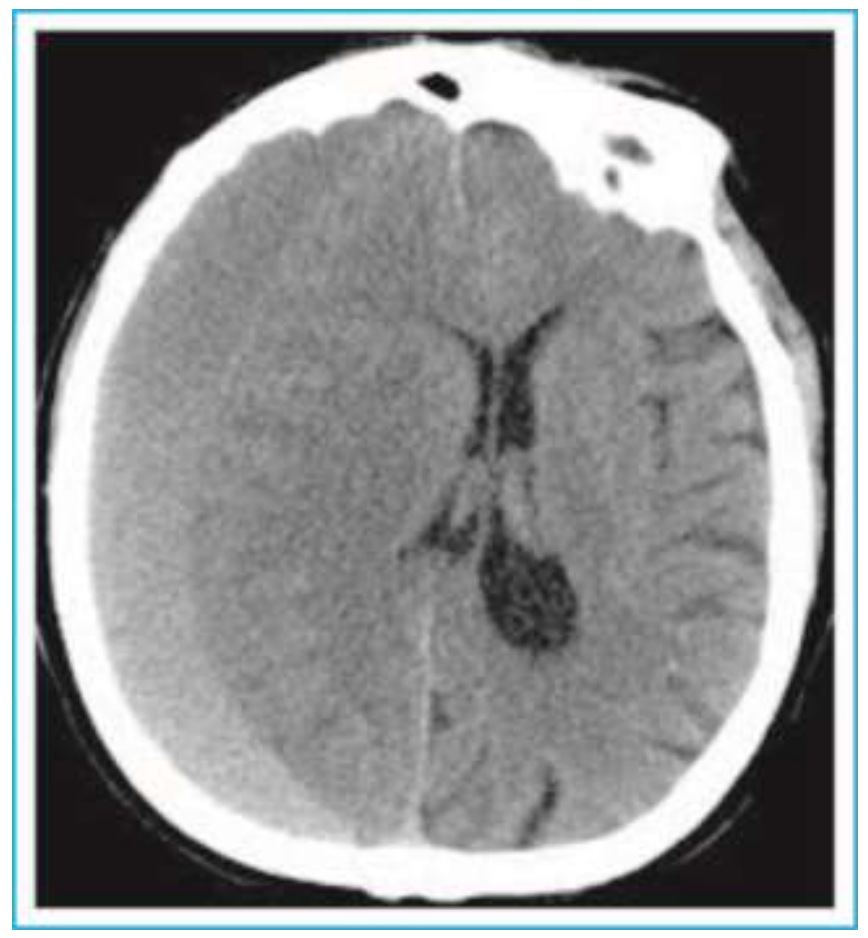

Figure 1: Right Fronto- parietal Chronic Subdural Hematoma with Acute Component.

\section{DISCUSSION}

Chronic subdural hematoma is a collection of liquefied blood between Dura and the arachnoid layer of Brain. Mostly patients present after three weeks of injury. ${ }^{9}$ Extent of surgery is still controversial. Burrhole craniotomy with subdural drain is yet is a treatment of choice; it is 
associated with low recurrence rate and complications. The evacuation of chronic subdural hematoma through single burrhole and closed drainage system under local anesthesia is safe and effective treatment modality for the management of chronic subdural hematoma. Single burrhole craniotomy for evacuation chronic subdural hematoma had lower or same recurrence rate as compared to two burr hole evacuation of chronic subdural hematoma. ${ }^{10}$ In our study, male and female ratio was 25:5 and 5:1 respectively. Likewise, in most of the national and international studies, male predominant is reported as Achakzai et al. reported in their 10year study research related to the surgical management of chronic subdural hematoma. ${ }^{8}$ Whereas, the age range was 50 to 80 years. Sarwar et al. also documented mean age 68 years. $^{11}$ In previous study, most common symptom was gait disturbance followed by one side weakness (Hemiparesis) in 17 patients (56.66\%), whereas, in our study, the headache was present in 11 patients (36.6\%), followed by dementia in 10 patients (33.33\%), incontinence in 7 patients (23.3\%), consciousness disturbance in 6 patients (20\%), vomiting in 3 patients (10\%), Seizures in 3 patients (10\%) and motor aphasia in 2 patients (6\%) patients. Similarly, Rashid et al. reported Hemiparesis in most of their patients of chronic subdural hematoma. ${ }^{12}$.

Our cure rate in chronic subdural hematoma after burrhole craniotomy with subdural drain was high. Post operatively, only two patients presented with recurrence of CSDH. Kitya et al. reported through a large series of 205 patients and concluded the same that burrhole surgical evacuation is a safe and reliable intervention in chronic subdural hematoma. ${ }^{13}$ Fortunately, not a single patient had been complicated with tension pneumocephalus. However, following are the associated risk factors, hypertension, chronic liver disease, use of anti-coagulants patients \& Diabetes mellitus. In our setting as well as Rovlias and Theodoropoulos reported, that due to increase in life expectancy, mostly the elder age group was on antiplatelet due to risk of strokes and DVT. So, these patients can be on a risk of development of chronic subdural hematoma in elderly population. ${ }^{14}$

\section{Recommendations}

Chronic subdural hematoma was found common in clinical entity in the neurosurgery department. Single burrhole craniotomy with close drainage system is a simple and safe procedure under local anesthesia for treatment of chronic subdural hematoma. Surgical outcome was high, which mostly depends on the preoperative neurological status and co morbidity factors.

\section{CONCLUSION}

Chronic subdural hematoma can be safely done under local anesthesia to avoid the hazards of general anesthesia and single burrhole aspiration with the close drainage system has a good surgical outcome. After evacuation of chronic subdural hematoma, cavity filled with normal saline to avoid pneumocephalus. Post-operative acute subdural hematoma should be avoided by proper hemostasis.

\section{REFERENCES}

1. Sapkota SN. Comparative Study of Surgical Outcome of Chronic Subdural Hematoma Treated with and without External Drainage. Eastern Green Neurosurgery, 2020; 2 (1): 30-34.

2. Rashid, S. M., Deliran, S. S., Dekker, M. C. J., \& Howlett, W. P. Chronic subdural hematomas: a case series from the medical ward of a north Tanzanian referral hospital. Egyptian Journal of Neurosurgery, 2019; 34 (1): 29.

3. Edlmann, E., Giorgi-Coll, S., Whitfield, P. C., Carpenter, K. L., \& Hutchinson, P. J. Pathophysiology of chronic subdural haematoma: inflammation, angiogenesis and implications for pharmacotherapy. Journal of neuroinflammation, 2017; 14 (1): 1-13. 
4. Yadav YR Chronic subdural. hematoma. Asian journal of neurosurgery, 2016: 330.

5. Das SS. Surgical Outcome of Chronic Subdural Haematoma: An Analysis of 300 Cases. Journal of Dhaka Medical College, 2015; 24 (2): 126-131.

6. Kolias AG. Chronic subdural haematoma: modern management and emerging therapies. Nature Reviews Neurology, 2014; 10 (10): 570-578.

7. Chen FM. Predictors of acute intracranial hemorrhage and recurrence of chronic subdural hematoma following burr hole drainage. BMC Neurology, 2020; 20 (1): 1-10.

8. Achakzai NU, Adil I, Khan S. Outcome of surgical management of chronic subdural hematoma. Pakistan Journal of Neurological Surgery, 2018; 22 (2): 61-66.

9. Asif $M$, Younus A. Chronic Subdural Hematoma, Review of 30 Cases Managed at DHQ Teaching Hospital, Sahiwal Medical College, Sahiwal. Pakistan Journal of Neurological Surgery, 2018; 22 (2): 67-70.

10. Yadav YR, Parihar V, Namdev H, Bajaj J. Chronic subdural hematoma. Asian Journal of Neurosurgery, 2016; 11 (4): 330.
11. SARWAR MA, Ali K, Hussain K, Shahid S, Abid M, Kazmi AR., et al. Management and Outcome of Chronic Subdural Hematoma: A Prospective Study of Shaikh Zayed Hospital, Lahore. Pakistan Journal of Neurological Surgery, 2018; 22 (1): 24-32.

12. Rashid SM, DeliranSS, Dekker MC. Howlett WP. Chronic subdural hematomas: a case series from the medical ward of a north Tanzanian referral hospital. Egyptian Journal of Neurosurgery, 2019; 34 (1): 29.

13. Kitya $D$, Punchak $M$, Abdelgadir J, Obiga O, Harborne D, Haglund MM. Causes, clinical presentation, management, and outcomes of chronic subdural hematoma at Mbarara Regional Referral Hospital. Neurosurgical Focus, 2018; 45 (4): E7.

14. Rovlias A, Theodoropoulos S, Papoutsakis D. Chronic subdural hematoma: surgical management and outcome in 986 cases: a classification and regression tree approach. Surgical Neurology International, 2015: 6.

\section{Additional Information}

Disclosures: Authors report no conflict of interest.

Ethical Review Board Approval: The study was conformed to the ethical review board requirements.

Human Subjects: Consent was obtained by all patients/participants in this study.

\section{Conflicts of Interest:}

In compliance with the ICMJE uniform disclosure form, all authors declare the following:

Financial Relationships: All authors have declared that they have no financial relationships at present or within the previous three years with any organizations that might have an interest in the submitted work.

Other Relationships: All authors have declared that there are no other relationships or activities that could appear to have influenced the submitted work. 
AUTHORS CONTRIBUTIONS:

\begin{tabular}{|l|l|l|}
\hline Sr.\# & Author's Full & Intellectual Contribution to Paper in Terms of: \\
\hline 1. & Mukhtiar Ahmed & 1. Basic study design and manuscript writing \\
\hline 2. & Fauzia Sajjad & 2. Analysis of data \\
\hline 3. & Ajmal Khan & 3. Data analysis \\
\hline 4. & Talha Abass & 4. Methodology \\
\hline 5. & Hamid Akbar & 5. Literature Review \\
\hline 6. & Khawar Anwar & 6. Literature Review \\
\hline
\end{tabular}

